\title{
OPTIMAL PILOT PLACEMENT FOR CHANNEL TRACKING IN OFDM
}

\author{
Min Dong and Lang Tong \\ School of Electrical and Computer Engineering \\ Cornell University, Ithaca, NY 14853 \\ \{mdong,ltong\}@ee.cornell.edu
}

\author{
Brian M. Sadler \\ Army Research Laboratory \\ Adelphi, MD 20783 \\ bsadler@arl.army.mil
}

\begin{abstract}
The problem of optimally placing pilot symbols for tracking frequency selective fading channels in an OFDM system is considered. The time-varying channel is approximated as a Gauss-Markov process. The Kalman filter, as the optimal tracking algorithm, is used for updating channel states in frequency. For a fixed percentage of pilot symbols, assuming pilot clusters are periodically sent through $(L+1)$ OFDM subchannels for training, we optimize the cluster size by minimizing the maximum steady-state mean square error (MSE) of the channel estimator. It is shown that single pilot periodic placement achieves the minimum MSE.
\end{abstract}

\section{INTRODUCTION}

Modern wireless communications require high data rate transmission over mobile channels. Multipath propagation causes intersymbol interference (ISI). Orthogonal Frequency Division Multiplexing (OFDM) is an attractive modulation technique to overcome the effect of ISI for high data rate applications, such as Digital Video Broadcast (DVB) and Digital Audio Broadcast (DAB). In a mobile scenario, due to Doppler spread, the channel is time-varying. Channel estimation over time-varying channels becomes critical to achieve reliable communication. To facilitate channel state acquisition, pilot symbols are usually inserted in the data stream. However, inserting training reduces the information transmission rate. Thus, a low percentage of training for channel tracking is desired, and the placement of these pilot symbols in the data stream for high quality tracking performance becomes an important issue. This paper focuses on the design of pilot symbol placement for channel tracking in an OFDM system.

The optimized placement of training symbols has been shown to enhance overall system performance from

This work was supported in part by the Army Research Office under Grant ARO-DAAB19-00-1-0507 and the Multidisciplinary University Research Initiative (MURI) under the Office of Naval Research Contract N00014-00-1-0564. both information and estimation theoretical perspectives $[1,4,2,8]$. For OFDM systems, the optimal placement of training for block fading channels are found for maximizing channel capacity [1], and the placement of superimposed training minimizing MSE of least-squares channel estimator is obtained in [9]. In [8], the placement of pilot symbols within an OFDM block is optimized with respect to the mean square error (MSE) of the channel estimate for a time-invariant channel. Based on this, two tracking schemes are then compared for time-varying channels for different degrees of channel state variation.

The optimal placement gain is especially significant for time-varying channels. In $[3,10,7]$, pilot placements in an infinite data stream are considered. [3] optimizes the placement by minimizing MSE of channel estimators using two tracking algorithms. [10] obtains the optimal spacing of pilots and power allocation at high SNR by maximizing a lower bound on capacity. In [7], the pilot symbols are inserted periodically and the optimal spacing between symbols maximizing the mutual information with binary input is calculated numerically. In [5], optimal training placement in a finite data packet is investigated to minimize the maximum MSE of the MMSE channel estimator for Rayleigh fading channel.

In this paper, we investigate the pilot placement for tracking frequency selective fading channels in an OFDM system. The time-varying channel is approximated as a Gauss-Markov process. We assume that $(L+1)$ OFDM subchannels are dedicated for training, where pilot symbol clusters are periodically sent. For a fixed percentage of pilots, the placement is characterized by the pilot cluster size. The Kalman Filter is used as the optimal tracking algorithm for updating channel states in frequency. We optimize the placement of pilot symbols by minimizing the maximum steady-state MSE of the channel estimator. It is shown that single pilot periodic placement achieves the minimum MSE. 


\section{PROBLEM STATEMENT}

\subsection{OFDM system for fading channels}

\subsubsection{The OFDM system}

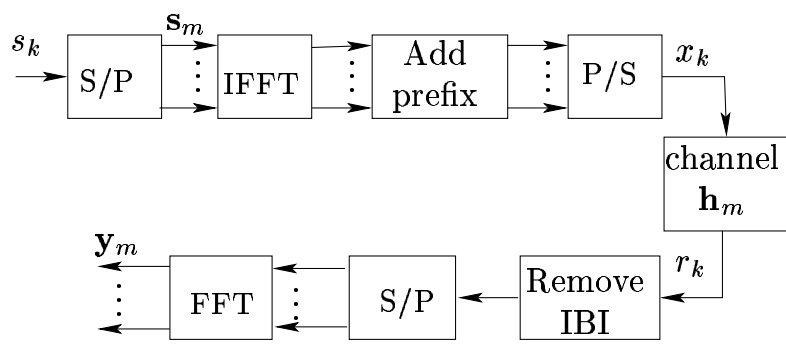

Fig. 1: A OFDM system

We consider an OFDM system with $N$ subchannels. The equivalent discrete-time baseband model is shown in Figure 1. The incoming data stream $\left\{s_{k}\right\}_{k=1}^{\infty}$ consists of independent and identically distributed (i.i.d.) symbols. The symbol stream is converted into blocks of parallel symbols by the serial to parallel converters (S/P). Each of these OFDM blocks consists of $N$ symbols that are carried on $N$ frequency subchannels, respectively. These blocks are then transformed by an $N$-point IFFT, and the cyclic prefix (CP) of length $L$ is appended to each of them. The newly formed blocks are then converted into a single data stream by a parallel to serial $(\mathrm{P} / \mathrm{S})$ converter, and transmitted over the channel.

\subsubsection{The fading channel model}

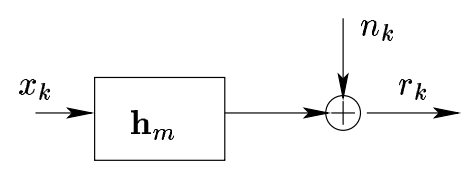

Fig. 2: A time-varying system model

We consider a slowly time-varying system shown in Figure 2. The channel $\mathbf{h}_{m}=\left[h_{m}[0], \cdots, h_{m}[L]\right]^{t}$ is assumed to be a frequency selective Rayleigh fading channel with order $L$. We assume that the channel taps $\left\{h_{m}[l]\right\}_{l=0}^{L}$ are i.i.d. Gaussian with zero mean and variance $\sigma_{h}^{2}$. The additive white noise $\mathbf{n}_{m}$ is assumed complex circular Gaussian and independent of $\mathbf{h}_{m}$.

We assume that the channel fading is relatively slow compared to the symbol rate, such that the channel state remains unchanged over the duration of each OFDM block, but changes from block to block. A first-order Gauss-Markov model can adequately characterize the dynamics of the slowly time-varying channel $[11,6]$. We consider the channel as the following first-order GaussMarkov process:

$$
\mathbf{h}_{m}=a \mathbf{h}_{m-1}+\mathbf{u}_{m}, \quad m=1,2, \cdots
$$

where $\mathbf{h}_{m} \sim \mathcal{C N}\left(0, \sigma_{h}^{2} \mathbf{I}\right)$, and $a$ is the fading correlation coefficient that may vary between zero and one according to the fading channel bandwidth $f_{d}$, i.e., Doppler spread. For transmission over bandwidth $W$ and decorrelation level $\phi$ that defines the coherence time $T_{c}\left(=1 / f_{d}\right), a$ is determined by $a^{\frac{W}{f_{d}}}=\phi$. Finally, $\mathbf{u}_{m} \stackrel{\text { i.i.d. }}{\sim} \mathcal{C N}\left(0, \sigma_{u}^{2} \mathbf{I}\right)$ is the driving noise with $\sigma_{u}^{2}=\left(1-a^{2}\right) \sigma_{h}^{2}$.

The system equation for each OFDM block can be written as

$$
\mathbf{y}_{m}=\mathbf{D}_{m} \mathbf{s}_{m}+\mathbf{n}_{m}, \quad m=1,2, \cdots
$$

where $\mathbf{s}_{m}, \mathbf{y}_{m}$ and $\mathbf{n}_{m}$ are $N$-by- 1 vectors representing the $m$-th transmitted data block, received signal and additive noise, respectively. $\mathbf{D}_{m}=\operatorname{diag}\left(d_{m, 1}, \cdots, d_{m, N}\right)$ is a $N$-by- $N$ diagonal matrix, where $d_{m, n}$ is the OFDM channel response of the $n$-th subchannel for the $m$-th data block. In addition, $d_{m, n}$ is the $n$-th Fourier coefficient of the $N$-point FFT of the channel $\mathbf{h}_{m}$ :

$$
\mathbf{d}_{m}=\left[\begin{array}{c}
d_{m, 1} \\
d_{m, 2} \\
\vdots \\
d_{m, N}
\end{array}\right]=\sqrt{N} \mathbf{W}_{L} \mathbf{h}_{m},
$$

where $\mathbf{W}_{L}$ is the truncated unitary FFT matrix of size $N$-by- $(L+1)$, i.e., $\left[\mathbf{W}_{L}\right]_{n k}=\frac{1}{N} \exp \left(\frac{-j 2 \pi(n-1)(k-1)}{N}\right)$.

\subsection{Pilot placement schemes}

In an OFDM system, pilot symbols may be sent on selected subchannels for channel estimation. For a timevarying system, pilot symbols become especially important to achieve reliable transmission. Since high information transmission rate mandates low percentage of training, pilot symbols are inserted periodically in OFDM blocks to track the channel state. Our final objective is then to find, among all such schemes, a placement that optimizes the tracking performance.

We now formulate the problem of designing optimal placement of pilot symbols. Let $\hat{\mathbf{h}}$ be the channel estimate, and the channel MSE is defined as $E\left\{\|\hat{\mathbf{h}}-\mathbf{h}\|^{2}\right\}$. In [8], for channel estimation within one OFDM block, it is shown that in the absence of the noise, any $(L+1)$ of the $N$ subchannels can be used for training to recover the channel $\mathbf{h}$ exactly; and in the presence of noise, equally-spaced placement of the $(L+1)$ pilot symbols is optimal, i.e., obtains the MMSE estimate of the channel. Therefore, in considering the placement 
schemes, we have the following assumptions

A1) The percentage of pilot symbols in the data stream, denoted as $\eta$, is fixed.

A2) Each OFDM block size $N=k(L+1), k \geq 1$.

A3) $(L+1)$ out of $N$ subchannels are selected periodically as training subchannels from the set $\{i, i+$ $\left.\frac{N}{L+1}, \cdots, i+\frac{L N}{L+1}\right\}, i=0,1, \cdots, \frac{N}{L+1}-1$. During a training period, pilot symbols in each OFDM block, denoted as $\mathbf{s}_{p, m}=\left[s_{p, m}[0], \cdots, s_{p, m}[L]\right]^{t}$, are sent over the selected $(L+1)$ training subchannels.

A4) Pilot symbols have equal power, i.e., $\left|s_{p, m}[i]\right|^{2}=$ $\sigma_{p}^{2}$, for any $m$ and $i$.

Since the channel state changes from block to block, we consider the type of periodical placements with equal training cluster size, as shown in Figure 3. Each column represents an OFDM block. The black parts represent pilot symbols. The placement of pilot symbols can be characterized by the pilot cluster length $r$. For each training subchannels, the pilot clusters are inserted at the beginning of each transmission period of length $T$. For fixed pilot percentage $\eta$, it is not hard to see that given the pilot cluster $r$, the transmission period length $T$ is then given by $T=\frac{(L+1) r}{N \eta}$. Note also that, when $r$ varies from $1 \rightarrow \infty$, the placement changes from the single pilot periodical placement to single cluster placement.

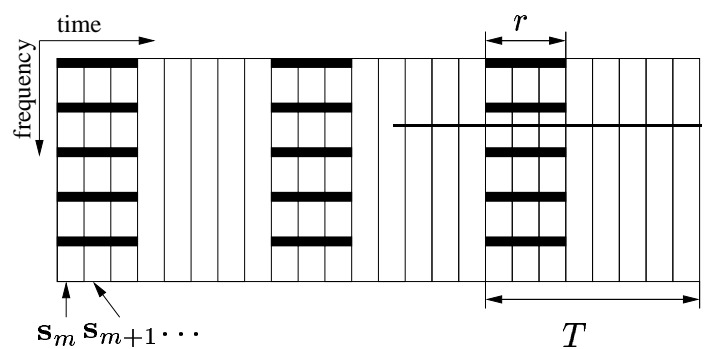

Fig. 3: Input OFDM blocks with training period $T$

\subsection{Channel tracking via Kalman filtering}

The problem of channel tracking has been widely studied. Among all linear tracking algorithms, the Kalman filter has long been known to be optimal in terms of minimizing the Bayesian MSE for each estimate of the channel state. In this paper, we are interested in optimizing the placement of pilot symbols under the Kalman filter channel tracking algorithm. The performance criterion is the steady-state maximum channel MSE during one period $T$, defined as

$$
\mathcal{E}(r)=\max _{1 \leq k \leq T} \lim _{l \rightarrow \infty} E\left\{\left\|\hat{\mathbf{h}}_{l T+k}-\mathbf{h}_{l T+k}\right\|^{2}\right\}
$$

and our objective is to find the optimal pilot cluster size $r$ that minimizes the maximum MSE of the estimator for OFDM channels

$$
r_{*}=\arg \min _{r} \mathcal{E}(r) .
$$

For the channel model we consider in (1), the timevarying OFDM subchannels and the received data satisfy the following state space model

$$
\begin{aligned}
\mathbf{d}_{m} & =a \mathbf{d}_{m-1}+\mathbf{W}_{L} \mathbf{u}_{m}, \\
\mathbf{y}_{m} & =\mathbf{S}_{m} \mathbf{d}_{m}+\mathbf{n}_{m}, \quad m=1,2, \cdots
\end{aligned}
$$

where $\mathbf{S}_{m}=\operatorname{diag}\left(\mathbf{s}_{m}\right)$. The state space model for the $(L+1)$ training subchannels is

$$
\begin{aligned}
\mathbf{d}_{p, m} & =a \mathbf{d}_{p, m-1}+\mathbf{W}_{p L} \mathbf{u}_{m}, \\
\mathbf{y}_{p, m} & =\mathbf{S}_{p, m} \mathbf{d}_{p, m}+\mathbf{n}_{p, m}, \quad m=1,2, \cdots
\end{aligned}
$$

where pilot matrix $\mathbf{S}_{p, m}=\operatorname{diag}\left(\mathbf{s}_{p, m}\right)$, and $\mathbf{W}_{p L}$ is the matrix $\mathbf{W}_{L}$ with selected rows according to the $(L+1)$ training subchannels. Note that by the equally-spaced selection of training subchannels, we have $\mathbf{W}_{p L} \mathbf{W}_{p L}^{H}=$ $\frac{L+1}{N} \mathbf{I}$. Therefore, the driving noise $\tilde{\mathbf{u}}_{m} \triangleq \mathbf{W}_{p L} \mathbf{u}_{m}$ is Gaussian with distribution $\mathcal{C N}\left(0, \frac{L+1}{N} \sigma_{u}^{2} \mathbf{I}\right)$, and the training subchannel $\mathbf{d}_{p, m} \sim \mathcal{C N}\left(0, \frac{L+1}{N} \sigma_{h}^{2} \mathbf{I}\right)$. This implies that the $(L+1)$ training subchannels are independent parallel channels, and the tracking in these channels can be performed individually.

Assume the fading correlation coefficient $a$ is known. Let $\left\{n_{0}, n_{1}, \cdots, n_{L}\right\}$ be the index set of training subchannels. The channel tracking using Kalman filtering is performed by the following two-step procedure:

1. During each training period, for the $(L+1)$ training subchannels, the receiver uses the Kalman filter update, yielding the MMSE update for each subchannel state

$$
M^{\left(n_{i}\right)}[l T+k]=\frac{\sigma_{n}^{2}\left(a^{2} M^{\left(n_{i}\right)}[l T+k-1]+\frac{L+1}{N} \sigma_{u}^{2}\right)}{\sigma_{n}^{2}+\left(a^{2} M^{\left(n_{i}\right)}[l T+k-1]+\frac{L+1}{N} \sigma_{u}^{2}\right) \sigma_{p}^{2}},
$$

for $k=1, \cdots, r$, where $M^{\left(n_{i}\right)}[l T+k]$ is the MMSE for the estimator of the $n_{i}$-th training subchannel for the $(l T+k)$-th OFDM block based on all the previous observations.

Denoting the channel estimator for the training subchannels as $\hat{\mathbf{d}}_{p, l T+k}$, the data subchannels can then be estimated as

$$
\hat{\mathbf{d}}_{d, l T+k}=\mathbf{W}_{d L} \mathbf{W}_{p L}^{-1} \hat{\mathbf{d}}_{p, l T+k} .
$$


for $k=1, \cdots, r$, where $\mathbf{W}_{d L}$ is the matrix $\mathbf{W}_{L}$ after deleting the $(L+1)$ selected rows corresponding to the training subchannels.

It is not hard to see that the MSE of each data subchannel $M^{(j)}[l T+k]\left(j \notin\left\{n_{i}\right\}_{i=0}^{L}\right)$ is equivalent to the MSE of each training subchannel $M^{\left(n_{i}\right)}[l T+k]$ in (10).

2. During the data transmission period, the receiver predicts the channel state based on the last Kalman filter update in the training period

$$
\hat{\mathbf{d}}_{l T+r+k}=a^{k} \hat{\mathbf{d}}_{l T+r}, \quad k=1, \cdots, T-r .
$$

From the relation between $\mathbf{h}_{l T+k}$ and $\mathbf{d}_{p, l T+k}$, it follows that $\mathcal{E}(r)$ in (4) can be rewritten as

$$
\begin{aligned}
\mathcal{E}(r) & =\max _{1 \leq k \leq T} \lim _{l \rightarrow \infty} E\left\{\left\|\mathbf{W}_{p L}^{-1} \hat{\mathbf{d}}_{p, l T+k}-\mathbf{W}_{p L}^{-1} \mathbf{d}_{p, l T+k}\right\|^{2}\right\} \\
& =\max _{1 \leq k \leq T} \lim _{l \rightarrow \infty} E\left\{\left\|\hat{\mathbf{d}}_{p, l T+k}-\mathbf{d}_{p, l T+k}\right\|^{2}\right\} \\
& =\max _{1 \leq k \leq T} \lim _{l \rightarrow \infty} \sum_{i=0}^{L} M^{\left(n_{i}\right)}[l T+k] .
\end{aligned}
$$

\section{OPTIMAL PLACEMENT FOR CHANNEL TRACKING}

For the $n_{i}$-th subchannel, the prediction MMSE during the data transmission period is

$$
\begin{array}{r}
M^{\left(n_{i}\right)}[l T+r+k]=a^{2} M^{\left(n_{i}\right)}[l T+r+k-1]+\frac{L+1}{N} \sigma_{u}^{2} \\
=a^{2 k} M^{\left(n_{i}\right)}[l T+r]+\frac{L+1}{N} \sigma_{u}^{2} \sum_{i=0}^{k-1} a^{2 i}, \\
\quad \text { for } k=1, \cdots, T-r . \quad
\end{array}
$$

As we have mentioned in the last section, elements in $\mathbf{d}_{p, l T+k}$ are i.i.d., and it follows that $M^{\left(n_{i}\right)}[l T+r]$ are all equivalent for $i=0, \cdots, L$. Then, the steady-state maximum MSE during one transmission period is

$$
\begin{aligned}
& \mathcal{E}(r)=\max _{1 \leq k \leq T} \lim _{l \rightarrow \infty}(L+1) M^{\left(n_{0}\right)}[l T+k]=(L+1) M_{T}^{\left(n_{0}\right)} \\
& =(L+1)\left(\frac{L+1}{N} \sigma_{h}^{2}-a^{2 r \frac{L+1-N \eta}{N \eta}}\left(\frac{L+1}{N} \sigma_{h}^{2}-M_{r}^{\left(n_{0}\right)}\right)\right) .
\end{aligned}
$$

where $M_{k}^{\left(n_{0}\right)}=\lim _{l \rightarrow \infty} M^{\left(n_{0}\right)}[l T+k]$ is the steadystate MSE of the $n_{0}$-th subchannel over the $k$ th OFDM block of a transmission period.

To obtain the steady-state MMSE $M_{T}^{\left(n_{0}\right)}$, we first see that if we only send pilots on this subchannel, then the channel state MSE under the Kalman filter converges to the steady-sate MSE, denoted as $M_{\infty}$. Its expression can be obtained from the standard steadystate Ricatti equation. Now, let

$$
\delta_{k}=M_{k}^{\left(n_{0}\right)}-M_{\infty} .
$$

From (10), it can be shown that, during a training period, i.e., $1 \leq k \leq r$,

$$
\delta_{k+1}=\frac{\delta_{1}}{1+\beta \frac{\delta_{1}}{\alpha-1}\left(1-\frac{1}{\alpha^{k}}\right)}\left(\frac{1}{\alpha}\right)^{k},
$$

where

$\alpha=\frac{1}{a^{2}}\left(1+\left(\left(1-a^{2}\right) \frac{L+1}{N} \sigma_{h}^{2}+a^{2} M_{\infty}\right) \frac{\sigma_{p}^{2}}{\sigma_{n}^{2}}\right)^{2}$
$\beta=\frac{\sigma_{p}^{2}}{\sigma_{n}^{2}}\left(1+\left(\left(1-a^{2}\right) \frac{L+1}{N} \sigma_{h}^{2}+a^{2} M_{\infty}\right) \frac{\sigma_{p}^{2}}{\sigma_{n}^{2}}\right)$.

And, during a data transmission period, i.e., $r \leq k \leq$ $T$,

$$
\delta_{k+1}=a^{2} \delta_{k}+\left(1-a^{2}\right)\left(\frac{L+1}{N} \sigma_{h}^{2}-M_{\infty}\right) .
$$

At steady-state, $\delta_{r}$ and $\delta_{T}$ satisfy the following equality

$$
\left\{\begin{array}{l}
\delta_{r}=\frac{\delta_{T}}{1+\beta \frac{\delta_{T}}{\frac{\alpha}{T}\left(1-1-\frac{1}{\alpha^{k}}\right)}}\left(\frac{1}{\alpha}\right)^{k}, \\
\delta_{T}=a^{2 r \frac{L+1-N \eta}{N \eta}} \delta_{r}+\left(1-a^{2 r \frac{L+1-N \eta}{N \eta}}\right)\left(\frac{L+1}{N} \sigma_{h}^{2}-M_{\infty}\right) .
\end{array}\right.
$$

Then, the steady-state expression for $\delta_{T}$ can be obtained. Thus we obtain the expression for $M_{T}^{\left(n_{0}\right)}$, and therefore, $\mathcal{E}(r)$. that

Our final goal is to find the optimal value $r_{*}$ such

$$
r_{*}=\arg \min _{r} \mathcal{E}(r) .
$$

We have the following theorem.

Theorem 1 Assume $\eta$ is fixed. For tracking the timevarying channel modeled in (1) in an OFDM system using the Kalman filter, the single pilot periodical placement is optimal, i.e.

$$
\begin{aligned}
r_{*} & =1 . \\
T_{*} & =\frac{L+1}{N \eta}
\end{aligned}
$$

The minimum $\mathcal{E}(r)$ is given by

$\mathcal{E}\left(r_{*}\right)=(L+1)\left(\frac{L+1}{N} \sigma_{h}^{2}-a^{2 \frac{L+1-N \eta}{N \eta}}\left(\frac{L+1}{N} \sigma_{h}^{2}-M_{1}^{\left(n_{0}\right)}\right)\right)$.

Theorem 1 indicates that sending pilot symbols frequently through training subchannels is a better scheme than sending larger pilot clusters with low training frequency. The former gives better channel state tracking performance. Furthermore, this optimality holds regardless of the value of $a, S N R$ and $\eta$. 


\section{NUMERICAL RESULTS}

We compared the estimation performance under different pilot placement schemes. The channel with order $L=4$ is Gaussian with variance $\sigma_{h}^{2}=1$. The OFDM block length $N=64$. The percentage of pilot symbols in the data stream is $6.25 \%$. Figure 4 shows the maximum channel MSE vs. channel correlation coefficient $a$ at $S N R=30 d B$ under different pilot cluster length $r$. For $a=0$ and $a=1$, as we expected, there is no gain given by the optimal placement. When $a$ falls between 0 and 1 , we observe that a significant gain of the tracking performance can be obtained by optimally placing pilot clusters.

Figure 5 shows the variation of the maximum MSE $\mathcal{E}(r)$ under $S N R$. We chose $a=0.95$ (for bandwidths in the range of $10 \mathrm{kHz}$ and Doppler spread of order $100 \mathrm{~Hz}$ [7] ). Again, we see that a large gain is obtained under the optimal placement.

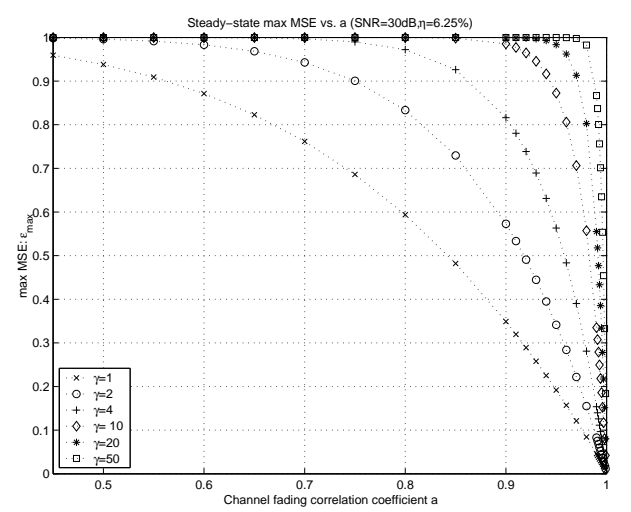

Fig. 4: $\mathcal{E}(r)$ vs. $a$ under different $r . N=64, L=4$. $S N R=30 d B, \eta=6.25 \%$.

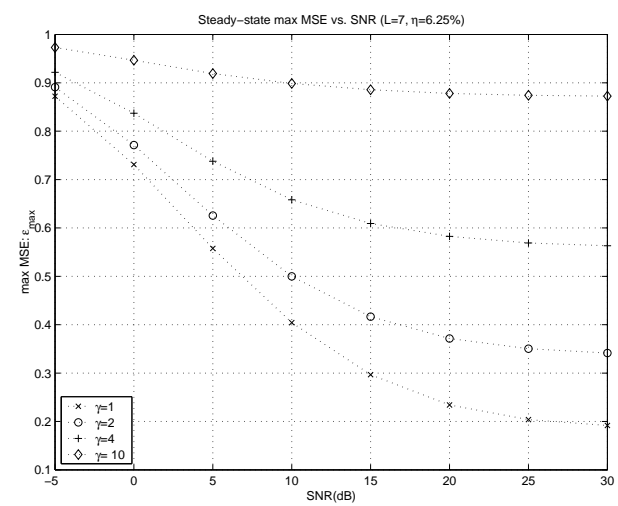

Fig. 5: $\mathcal{E}(r)$ vs. $S N R$ under different $r . N=64$, $L=4$. $a=0.95, \eta=6.25 \%$.

\section{CONCLUSION}

In this paper, we considered the problem of optimal placement of pilot symbols for tracking fading channels in an OFDM system. The first-order Gauss-Markov model is used to represent the dynamics of the fading channel. We choose $(L+1)$ equally-spaced subchannels for training. Pilot symbols are sent through these training subchannels periodically. Under the Kalman filter update, we obtain the steady-state MSE of the OFDM subchannels during the training period. It is then shown that the optimal placement is to send a single pilot periodically on each training channel. This allows for better tracking of the channel state variation.

\section{REFERENCES}

[1] S. Adireddy, L.Tong, and H.Viswanathan. "Optimal Placement of Training for Frequency Selective BlockFading Channels". IEEE Trans. on Info. Theory, 48(8), August 2002.

[2] B.Hassibi and B.Hochwald. "How much Training is Needed in Multiple-Antenna Wireless Links". Submitted to IEEE Trans. Information Theory, August 2000.

[3] M. Dong and L. Tong. "Optimal Placement of Training for Channel Estimation and Tracking". In MILCOM, Vienna, Virginia, October 2001.

[4] M. Dong and L. Tong. "Optimal Design and Placement of Pilot Symbols for Channel Estimation". IEEE Trans. on Signal Processing, 50(12):3055-3069, December 2002.

[5] M. Dong, L. Tong, and B. M. Sadler. "Training Placement for Tracking Fading Channels". In ICASSP, May 2002.

[6] T. Eyceoz, A. Duel-Hallen, and H. Hallen. "Deterministic channel modeling and long range prediction of fast fading mobile radio channels". IEEE Trans. Communications Letters, 2(9):254 -256, Sept. 1998.

[7] M. Medard, I. Abou-Faycal, and U. Madhowf. "Adaptive Coding with Pilot Signals". In 38th Allerton Conference, October 2000.

[8] R. Negi and J. Cioffi. "Pilot Tone Selection For Channel Estimation In A Mobile OFDM System". IEEE Trans. on Consumer Electronics, 44(3):1122-1128, Aug. 1998.

[9] S. Ohno and G. B. Giannakis. "Optimal training and redundant precoding for block transmissions with application to wireless OFDM". In ICASSP, May 2001.

[10] S. Ohno and G. B. Giannakis. "Average-Rate Optimal PSAM Transmissions over Time-Selective Fading Channels". IEEE Trans. on Wireless Communications, 1:712-720, October 2002.

[11] P.H.-Y. Wu and A. Duel-Hallen. "Multiuser detectors with disjoint Kalman channel estimators for synchronous CDMA mobile radio channels". IEEE Trans. Commun., 48(5):752-756, May 2000. 